\title{
What do exotic equations of state have to offer?
}

\author{
J.E. Horvath \\ J.E. Horvath \\ IAG, Department of Astronomy \\ Universidade de São Paulo, \\ Rua do Matão 1226, 05508-900 São Paulo SP, Brazil \\ Tel.: +55-11-30912806 \\ foton@astro.iag.usp.br
}

\begin{abstract}
We present a short general overview of the main features of exotic models of neutron stars, focusing on the structural and dynamical predictions derived from them. In particular, we discuss the presence of "normal" quark matter and ColorFlavor Locked (CFL) states, including their possible self-bound versions, and mention some different proposals emerging from the study of QCD microphysics. A connection with actual observed data is the main goal to be addressed at this talk and along the meeting. It is demonstrated that exotic equations of state are not soft if the vacuum contributions are large enough, and argued that recent measurements of high pulsar masses $\left(M \geq 2 M_{\odot}\right)$ create problems for hadronic models in which hyperons should be present.
\end{abstract}

\section{Introduction}

The quest of the internal composition of compact stars has been going on for decades, in close connection with the work in nuclear and particle physics. Although deemed in some sense "simpler" than magnetospheric phenomena, dealing with matter at the extreme conditions inside pulsars/neutron stars has never been easy. As 2006, the consensus about the nature of matter at several times the nuclear saturation density is weak, if anything. Several phases/components of the nuclear fluid have been proposed and studied, but decisive evidence for or against them is hard to obtain. In fact, and as discussed several times during this meeting, advances on the observational side has allowed one for the first time to probe key macroscopic properties of compact stars (masses, radii and a few others) that reflect the internal composition indirectly. However, a few remarks on this last statement are in order: on the one hand the precision attained by measurements has improved greatly but 
not to the point far beyond any suspicion, and on the other hand, supranuclear components with poorly constrained parameters (coupling constants, vacuum expectation values, etc.) will not be ruled out, even by very precise observations. Therefore, work is needed on the theoretical and terrestrial laboratories as well.

Overall, I believe it is fair to state that the bulk structural properties are well-known in the subnuclear domain (more strictly, below the neutron drip density $\sim 10^{11} \mathrm{~g} \mathrm{~cm}^{-3}$ ). However, important questions involving magnetic fields still remain (van Adelsberg et al. 2005). This is quite important to settle since it is where the star surface is seen by experiments due to electromagnetic radiation (see discussions about spectral lines and related topics in this meeting). There is also important information in timing irregularities, most notably glitches, believed to originate at the inner crust (although the "conventional wisdom" has been recently challenged, see the contribution by B. Link in this meeting and references therein) However, deep below the stellar crust it is increasingly difficult to construct a clear picture of the composition and therefore of the stellar structure. Condensates $\left(\pi^{-}, K^{-}\right.$, etc) are still possible depending on microphysics and drastically alter the thermal and dynamical behavior of the star. Fundamental degrees of freedom (i.e. quarks) may also constitute the main part of the core, and even most of the star if they happen to be of the "self-bound" type (i.e. do not decay back to ordinary nuclear species once formed). Recently, a lot of attention has been paid to paired quark matter in a variety of phases still being studied. A recent summary of these matters can be found in Weber (2005). Hereafter we shall mainly concentrate on the self-bound phases because they bring the greatest modifications to the structure and they remain viable alternatives to the supranuclear matter composition.

\section{Strange quark matter and its paired version}

Strange quark matter (SQM) is an extreme version of a cold quark plasma in which, by hypothesis, the energy per baryon number unit is selected (with the chosen parameters) to fall below the mass of the nucleon. This possibility of having matter so strongly bound that it does not wish to return to the normal hadronic state was first discussed by Bodmer (1971), rediscovered by Terazawa (1979) and finally relaunched colorfully by Witten (1984) more than 20 years ago. Many studies, both experimental and theoretical, devoted to the SQM hypothesis have produced interesting results and some controversial arguments against its existence, but with loopholes in them. A few candidates have appeared to SQM in cosmic rays (Björken \& McLerran 1979, Ichimura, Kamioka, Kitazawa et al. 1993, Choutko 2003, Madsen 2005) with fluxes consistent with astrophysical injection scenarios (i.e. merging of compact stars, supernovae). SQM formation on $\tau \leq 1 s$ timescale has been studied 
(Benvenuto \& Horvath 1989, Lugones, Benvenuto \& Vucetich 1994, Dai, Peng \& Lu 1995) and tentatively related to core-collapse supernovae, perhaps driven by photons (Chen \& Xu 2006) instead of mechanical energy transfer or neutrinos.

The existence of SQM would be important for compact objects, since within this picture all of them should be "strange stars" instead of neutron stars. However, there is still the issue of the timescale for the conversion, since while in supernovae models the latter is quite short, it could be stretched by several orders of magnitude depending on microphysical details (see, for example, Lugones \& Bombaci 2005). For the static, non-rotating structure, strange stars are constructed by integrating the Tolman-Oppenheimer-Volkoff equation with an equation of state of the form

$$
P=\frac{1}{3}(\rho-4 B)
$$

which has been extensively used because of its proximity with more detailed calculations including the finite s-quark mass and quark-quark interactions. The importance of the vacuum term, here written as $4 B$ in the spirit of the well-known MIT bag model (Degrand, Jaffe, Johnson \& Kiskis 1975) which produces a zero point pressure at finite (and large) energy density can not be overstated: the Bodmer-Witten-Terazawa hypothesis would preclude a "normal" matter crust in contact with it (thus limiting its total mass) unless a structured form of the quark matter itself is present (Benvenuto, Horvath \& Vucetich 1990, Heiselberg, Pethick \& Staubo 1993, Alford, Rajagopal, Reddy \& Steiner 2006) and may be responsible for phenomena commonly attributed to the inner crust (i.e. glitches). The alternative is a "floating" normal crust supported by electrostatic forces, and then necessarily quite light, perhaps too light to produce the observed phenomenology.

Quite independently of these considerations, there is a widespread belief that, because of its underlying free quark derivation, an equation of state (EOS) like eq.(1) must be very soft. This is far from being true: the issue of the softness/stiffness is rather related to the vacuum energy term, the real agent which determines the hardness of the EOS. We shall give examples of this behavior when discussing the stellar models.

A lot of activity has recently been seen on the effects of pairing interactions in dense quark matter. The issue is not new, since in the early ' 80 s a few works addressed the superfluid/superconducting properties of paired quarks (Bailin \& Love 1984 and references therein). However, those approaches were based on perturbative schemes, and therefore obtained (quite consistently) gaps of the order of $1 \mathrm{MeV}$ or so, much smaller than the natural scales of the problem (say, the quark chemical potential). The recent works (Alford \& Cowan 2006, Gómez Dumm, Blaschke, Grunfeld \& Scoccola 2006 and references therein) 
have tried to calculate the phase diagram more directly, without resorting to perturbative schemes. As a result, several pairing possibilities ( $u$ and $d$ quarks only, 2SC phase; all $u, d$ and $s$ quarks at a common Fermi momentum -not energy!-, the CFL state) were found with gaps as large as $100 \mathrm{MeV}$. Other possibilities, like a gapless CFL phase or the solidlike Larkin-Ovchinnikov-Fulde-Ferrell (LOFF) state are being considered and reflect a high complexity of the QCD phase diagram that might be important for compact stars (Ruster, Shovkovy \& Rischke 2004).

While this task continues, it is perhaps worthwhile to remark the importance of pairing energies for the stability issue discussed above: it is found in simple models (Lugones \& Horvath 2003) that the stability window (i.e. the place in parameter space inside which paired CFL matter would be stable) is greatly enhanced when compared with the same parameters for unpaired matter. Fig. 1 displays the situation for a model with constant gaps which were varied within the expected range. Perhaps pairing is a big clue to the ground state of matter relevant to astrophysics after all. This is the meaning of the (unimaginative) name "CFL strange matter".

\section{Effects on stellar models}

The general trend of stellar models calculated with self-bound equations of state is wellknown: in sharp contrast with neutron matter calculations, for which $R$ grows to $\sim 100 \mathrm{~km}$ for finite small baryonic mass values, self-bound stars can be found, in principle, down to tennis-ball sizes continuously (i.e. $R \rightarrow 0$ when $M \rightarrow 0$ ) and beyond, inside the realm of "strangelets". This is because binding comes from strong interactions and not from gravity. Of course, there is a Chandrasekhar mass for SQM or CFL strange matter sequences, which brings us to the question of the vacuum energy again.

In its simplest form of eq.(1) it is well-known that stellar models at the maximum mass scale as $B^{1 / 2}$. This property is related to the linearity of the EOS, and holds approximately if the latter is not strict. When pairing energy is present, the free energy of the paired mixture $\Omega_{C F L}$ is smaller than the unpaired version by a term quadratic both in the gap $\Delta$ and the chemical potential $\mu$ (Alford \& Reddy 2003)

$$
\Omega_{C F L}=\Omega_{\text {free }}-\frac{3}{\pi^{2}} \Delta^{2} \mu^{2}+B
$$

All the important thermodynamic quantities can be derived from eq.(2), which provides an equation of state for the CFL mixture. With that ingredient it is immediate to calculate stellar sequences of cold stars composed by this self-bound version of quarks. In this approach 
the gap has been assumed as a constant, in fact theoretical expectations strongly suggest that a functional dependence ensures, but at this time it is not possible to state anything reasonable and quantitative about its nature. The maximum mass along the sequence is shown in Fig. 2, and increases with increasing gaps. Of course, there must be an upper limit to the pairing energy gain in nature, otherwise all matter would decay into the more bound state (as pointed out by P. Haensel during the meeting), but this limit is not obvious and must be calculated consistently for each considered model (see Fig. 1). One important point to note here is the rather high values for the maximum mass along the sequence that can be obtained for gap values deemed quite modest (i.e. $M_{\max } \geq 2 M_{\odot}$ for $\Delta \sim 100 \mathrm{MeV}$ ). This means that the EOS is not soft, but rather stiff whenever the effective vacuum energy is large enough (composed in these models by a combination of the true vacuum and the condensation energy together).

With the use of analytical general relativistic solutions (Delgaty \& Lake 1998) one can go further and find the locus of mass maxima as a function of the radius $R_{\max }$. The answer is a curve indicating that, in general, larger maximum masses of self-bound sequences must have increasingly larger radii. Therefore, the observations of large compact star masses can potentially set a lower limit to their radii as well. This test is perhaps one of the simplest to perform since masses can be in some cases obtained with great precision, whereas radii are somewhat more indirect.

\section{Masses and radii: recent observations}

The zoo of compact star masses and radii has been growing recently, and there is now a firm expectation of finding more reliable limits to the internal structure than hitherto possible. From this point of view, important determinations are those of $M \gg 1.4 M_{\odot}$ and $M \ll 1.4 M_{\odot}$, because this are the limits where the self-bound and conventional models are more different. Nice, Splaver, Stairs et al.(2005) claim of $M=2.1 \pm 0.2 M_{\odot}\left(M=2.1_{0.5}^{0.4} M_{\odot}\right.$ at $2-\sigma$ level) for the compact star designated as PSR J0751+1807, and the Baker, Norton \& Quaintrell (2005) determination of $M=0.91 \pm 0.08 M_{\odot}$ for SMC X-1 are just two examples of this "spreading" around the older canonical value of $1.4 M_{\odot}$.

In fact this is one of the main reasons of why should we care about self-bound models: while it is generally believed that high masses disfavor a quark composition, it could be that hadronic models have a serious problem with them. This is because the appearance of hyperons (known to exist for decades) generally soften the equation of state below $1.4 M_{\odot}$ or so, a result found consistently over the years from microphysical approaches. The existence of quark cores does not help either because the maximum mass decreases with respect to 
the purely hadronic model. Therefore, either hyperons couple to neutrons and protons with strengths capable of giving a large extra repulsion (thus rising the maximum mass of the sequence), or it is acknowledged that truly exotic models, like the self-bound ones, are more compatible with the high masses (the problem with hyperons has been noted before by $\mathrm{M}$. Baldo, F. Burgio and coworkers). But this extreme possibility would also predict that the big difference in radii between conventional and self-bound models would begin below $0.5 M_{\odot}$, not around $1 M_{\odot}$, and it is unclear how and if such low-mass stars are formed in nature. This finally means that $\sim 1 M_{\odot}$ stars should show radii around $10 \mathrm{~km}$, not $6-7 \mathrm{~km}$ as previously thought. While we can not prove that exotic matter is present in compact stars, it is also not

guaranteed that microscopic EOS with all the degrees of freedom known from laboratories can fit the observations either.

\section{Conclusions}

We end this brief exposition about some features of self-bound models by saying that understanding of the vacuum is the real clue for advances in dense matter physics. This is not unlike other fields of physics, like the well-known crisis in cosmology about what the quantum vacuum should be and what actually is (Freedman \& Turner 2003). The same vacuum issue, but related to matter well above the saturation density, looks even more formidable, and its understanding should solve in the wash the issues of the existence of self-bound states and the features of the resulting EOS. It is also important to remark again that there is no hadronic model devoid of problems with the high mass end: either they ignore hyperons or are solved in a mean field approach or some other scheme with its own problems and questions. There is, however, the possibility of an extreme stiffness of the equation of state, such as the hyperons do not appear at all, because the relatively low density at the center. The good news is that we can now foresee actual tests of the stiffness of the EOS in the near future.

As stated, this stiffness is very important to establish, because the very introduction of $\Lambda$ particles and other hyperons would then call for very exotic interactions among them at least. Otherwise the resulting EOS become so soft that measured masses around $2 M_{\odot}$ happen to lie well above the maximum masses of the respective theoretical sequences. This is why strongly repulsive interactions would be required if hyperons appear inside compact stars. High masses may be pointing towards the exotica rather than excluding them (Özel 2006, Alford, Blaschke, Drago et al. 2006).

To conclude, we would like to quote an inspiring sentence from the English literature that may (or may not) be related to these topics, in which a bit of fantasy is always hidden 
Horatio, there are more things in Heaven and Earth

Than are dreamt of in your philosophy

Hamlet, Act I, Scene V

\section{Acknowledgements}

We would like to acknowledge the financial support of $\mathrm{CNPq}$ (Brazil) and the Organizing Committee for a great Workshop full of excellent presentations. I. Bombaci (U. of Pisa) is also acknowledged for extensive discussions and insightful ideas on dense matter that lead to improve this talk substantially. I also thank the warm hospitality and scientific advise of Dr. G. Lugones on these topics.

\section{REFERENCES}

Alford, M. \& Reddy, S., Phys. Rev. D 67, 074024 (2003)

Alford, M. \& Cowan, G., J.Phys.G 32, 511 (2006)

Alford, M.G., Rajagopal, K., Reddy, S. \& Steiner, A., Phys.Rev.D 73, 114016 (2006)

Alford, M., Blaschke, D., Drago, A., Klahn, T., Pagliara, G. \& Schaffner-Bielich, J., astro$\mathrm{ph} / 0606524$ (2006)

Bailin, D. \& Love, A., Physics Reports 107, 325 (1984)

Benvenuto, O.G. \& Horvath, J.E., Phys.Rev.Lett.63, 716 (1989)

Benvenuto, O.G., Horvath, J.E. \& Vucetich, H., Phys.Rev.Lett.64, 713 (1990)

Björken, J. D. \& McLerran, L. D., Phys.Rev.D 20, 2353 (1979)

Bodmer, A.R., Phys. Rev. D 4, 1601 (1971)

Chen, A. \& Xu, R.X., astro-ph/0605285 (2006)

Choutko, V. (for the AMS-01 Collaboration), In : Kajita, T., Asaoka, Y., Kawachi,A., Matsubara Y. and Sasaki, M. (eds.) Proceedings of the 28th International Cosmic Ray Conference, Tsukuba, Japan, July 31- August 7, 1765 Universal Academic Press, Tokyo (2003) 
Dai, Z.G., Peng, Q.H. \& Lu, T., ApJ. 440, 815 (1995)

Degrand, T., Jaffe, R.L., Johnson, K. \& Kiskis, J., Phys.Rev.D 12, 2060 (1975)

Delgaty, M.S.R. \& Lake, K., Comput.Phys.Commun.115, 395 (1998)

Freedman, W. L. \& Turner, M.S., Rev.Mod.Phys.75, 1433 (2003)

Gómez Dumm, D., Blaschke, D.B., Grunfeld, A.G. \& Scoccola, N.N., Phys. Rev.D 73, $114019(2006)$

Heiselberg, H., Pethick, C. J. \& Staubo, E. F., Phys.Rev.Lett.70, 1355 (1993)

Ichimura. M., Kamioka, E., Kitazawa, M. et al., Nuovo Cimento A 106, 843 (1993)

Lugones, G. \& Bombaci, I., Phys.Rev.D 72, 065021 (2005)

Lugones, G., Benvenuto, O.G. \& Vucetich, H., Phys. Rev.D 50, 6100 (1994)

Lugones, G. \& Horvath, J.E., A\&A 403, 173 (2003)

Lugones, G. \& Horvath, J.E., Phys.Rev.D.66, 074017 (2002)

Madsen, J., Phys.Rev.D 71014026 (2005)

Nice, D. , Splaver, E. M.; Stairs, I. H. et al., ApJ.634, 1242 (2005)

Özel, F., Nature 441, 1115 (2006)

Ruster, S.B., Shovkovy, I.A. \& Rischke, D.H., Nucl.Phys.A 743, 127 (2004)

Terazawa H., INS-Report 336 (1979)

Val Baker, A.K., Norton, A.J. \& Quaintrell, H., A\&A 441, 685 (2005)

van Adelsberg, M., Lai, Dong, Potekhin, A.Y. \& Arras, P., ApJ. 628, 902 (2005)

Weber, F., Prog.Part.Nucl.Phys. 54, 193 (2005)

Witten, E., Phys.Rev.D 30, 272 (1984) 


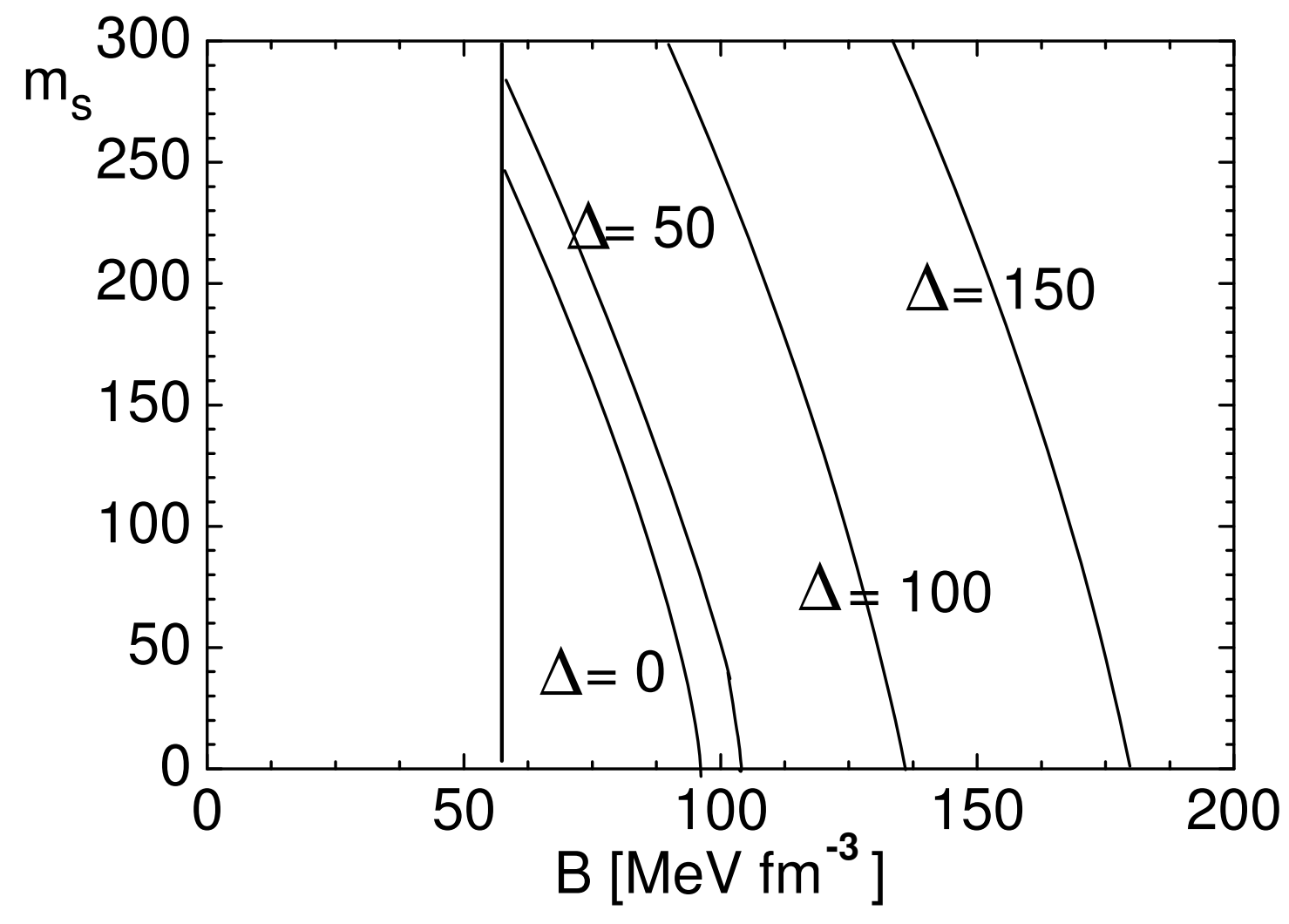

Fig. 1.- The stability windows for CFL strange matter. If the strange quark mass $m_{s}$ and the bag constant $B$ lie inside the bounded region the CFL state is absolutely stable. Each window is plotted for $P=0$, the stability window is the region between the vertical line (obtained by requiring instability of two-flavor quark matter) and the curve with a given value of the gap $\Delta$ as indicated by the label. Note the enlargement of the window with increasing $\Delta$. See Lugones \& Horvath (2002) for details. 


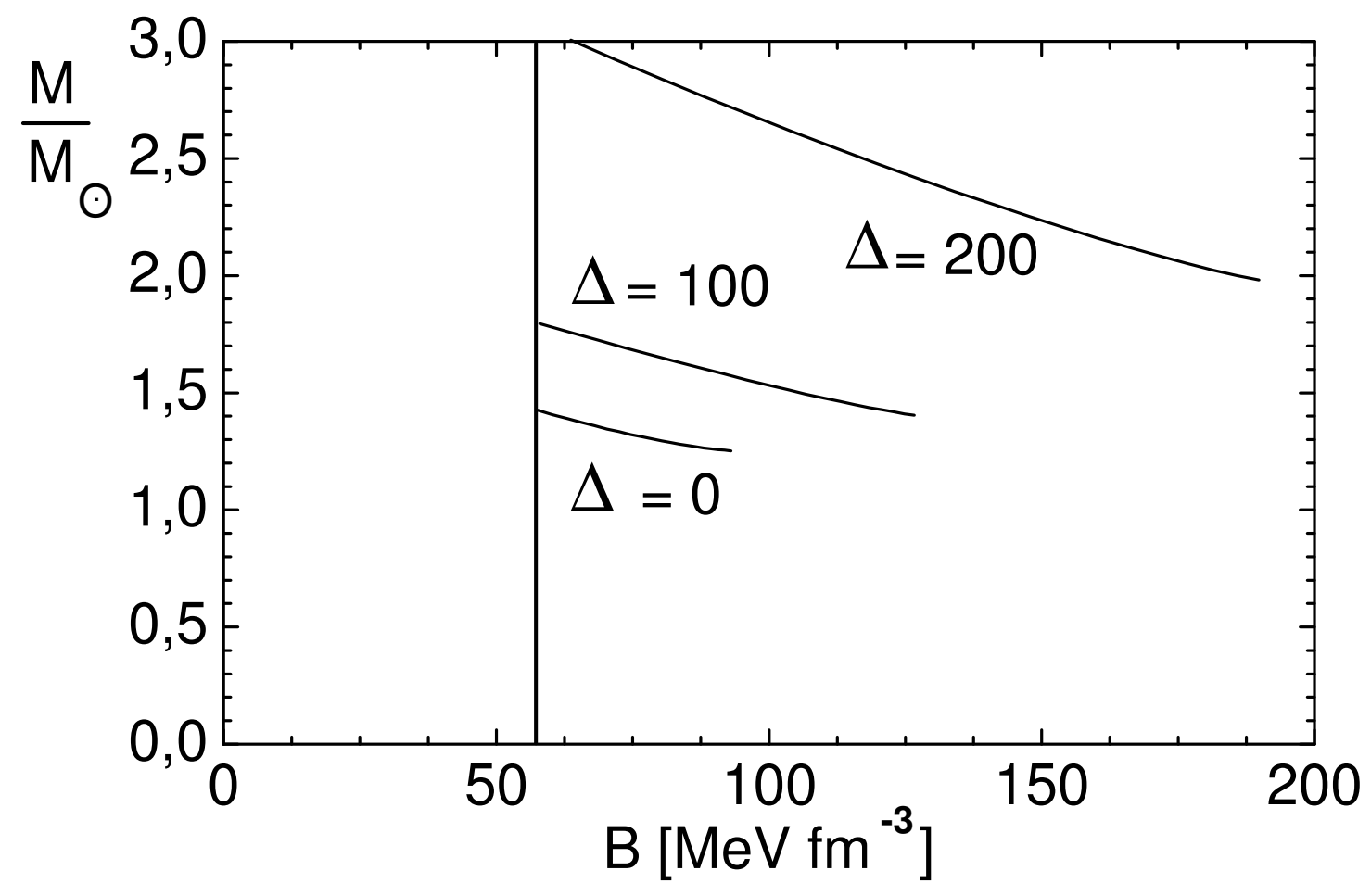

Fig. 2. - The maximum mass of each stellar sequence is shown here as a function of $B$, for $m_{s}=150 \mathrm{MeV}$ and different values of $\Delta$, the range of the latter is the same as in Fig. 1 . 